\title{
The Radial Velocity Projection Factor in the Pulsating Helium Star V652 Her
}

\author{
P. Montañés Rodriguez, C. S. Jeffery, R. Aznar Cuadrado \\ Armagh Observatory, College Hill, Armagh BT61 9DG, Northern \\ Ireland \\ D. Pollacco
}

Isaac Newton Group, La Palma, Spain

\begin{abstract}
The behavior of the velocity projection factor was studied for three individual Lines, He I $4438 \AA$, Si III $4553 \AA$, and N II $4442 \AA$, and for the complete spectrum in the radially pulsating helium rich star V652 Her. It is shown that the projection factor is itself a function of expansion velocity.
\end{abstract}

Apparent radial velocity, $v$, and the actual expansion velocity in radially expanding atmospheres $(\dot{r})$ are related by the projection factor $p=\dot{r} / v$ (Getting 1935, Parsons 1972). Parsons obtained $p=1.31 \pm 0.03$ for classical Cepheids, but this value may depend on several factors, including the limb-darkening in the line used to make the velocity measurement, the intrinsic profile of this line, and the method of measurement of the apparent radial velocity.

A new set of high S/N spectra of the radially pulsating, helium-rich star V652 Her (Hill et al. 1981) were obtained with the William Herschel telescope in 1998 July. They offered the opportunity to re-derive the atmospheric and pulsation properties with unprecedented precision.

From analyses of an average spectrum, other atmospheric properties of V652 Her have been found, including $T_{\text {eff }}=24550 \mathrm{~K}, \log g=3.7(\mathrm{cgs})$, chemical surface composition (98\% helium), and metallicity $(Z=0.015)$; these indicate that Z-bump opacities drive the pulsations (Jeffery, Hill, \& Heber 1999). In a previous radius measurement of V652 Her, Lynas-Gray et al. (1984) adopted $p=1.31$, but suggested that this assumption should be verified for hydrogenpoor atmospheres.

The behavior of $p$ was studied using an LTE model atmosphere to calculate synthetic spectra with the previous parameters and microturbulent velocity $v_{t}=5 \mathrm{~km} \mathrm{~s}^{-1}$. A grid of specific intensities $I(\lambda, \mu)$ as a function of wavelength $\lambda$ at a series of angles $\mu=\cos \theta$ (where $\theta$ is the angle between the line of sight and the normal to the atmosphere) was computed. A Doppler velocity shift was introduced to obtain the apparent intensity at limb angle $\mu$. The predicted emergent fluxes were obtained by integration over the disk: $F(\lambda)=2 \int_{0}^{1} I(\lambda, \mu) \mu \mathrm{d} \mu$. 

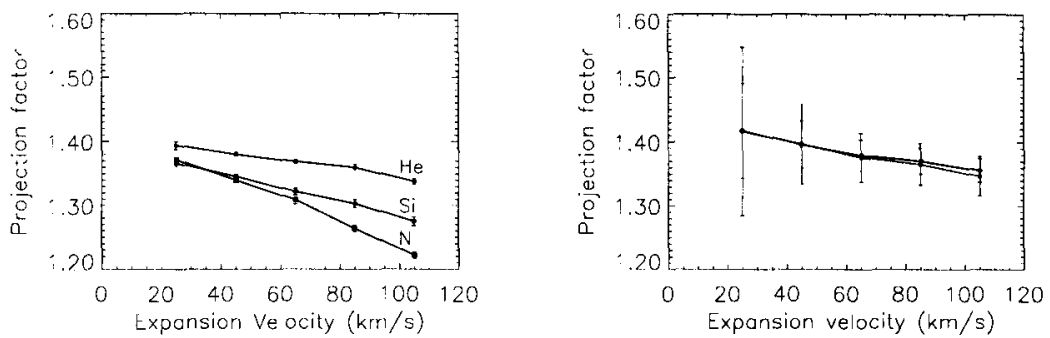

Figure 1. Relationship between the projection factor $p$ and the expansion velocity $\dot{r}$ in the case of individual lines (left) and for the complete spectrum excluding strong lines (right), without (lower line) and with rotation (upper line).

The synthetic spectra have been re-sampled with $\delta \lambda=0.223 \AA$ to make them directly comparable to the observed data.

We have shown that the projection factor is (a) a function of the expansion velocity, approximately linear, of the form $p=1.43-0.07 \dot{r} / 100$ (Figure 1), and (b) sensitive to the method of measurement. Our values for $p$ are up to $10 \%$ larger than that commonly used in the measurement of Cepheid radii.

For V652 Her, with $v_{\text {rot }} \sin i=21 \mathrm{~km} \mathrm{~s}^{-1}$, the inclusion of rotational broadening does not significantly alter the projection factor, although it does affect the resulting line widths. The results obtained are comparable with similar results for classical Cepheids obtained by Albrow \& Cottrell (1994) and others, and have important consequences for the determination of Cepheid radii.

\section{References}

Albrow, M. D. \& Cottrell, P. L. 1994, MNRAS, 267, 548

Getting, I. A. 1935, MNRAS, 95, 141

Hill, P. W., Kilkenny, D., Schönberner, D., \& Walker, H. J. 1981, MNRAS, 197, 81

Jeffery, C. S., Hill, P. W., \& Heber, U. 1999, A\&A, 346, 491

Lynas-Gray, A. E., Schönberner, D., Hill, P. W., \& Heber, U. 1984, MNRAS, 209,387

Parsons, S. B. 1972, ApJ, 174, 57 